\title{
Hadron spectroscopy from canonical partition functions
}

\author{
Z. Fodor ${ }^{a, b, c}$, K. K. Szabó ${ }^{a}$ and B. C. Tóth ${ }^{a, b}$ \\ ${ }^{a}$ Department of Physics, University of Wuppertal, Germany \\ ${ }^{b}$ Institute for Theoretical Physics, Eötvös University, Budapest, Hungary \\ ${ }^{c}$ Department of Physics, University of California, San Diego, USA
}

October 20, 2018

\begin{abstract}
A spectroscopic method for staggered fermions based on thermodynamical considerations is proposed. The canonical partition functions corresponding to the different quark number sectors are expressed in the low temperature limit as polynomials of the eigenvalues of the reduced fermion matrix. Taking the zero temperature limit yields the masses of the lowest states. The method is successfully applied to the Goldstone pion and both dynamical and quenched results are presented showing good agreement with that of standard spectroscopy. Though in principle the method can be used to obtain the baryon and dibaryon masses, due to their high computational costs such calculations are practically unreachable.
\end{abstract}

\section{Introduction}

One of the most critical steps in hadron spectroscopy is the choice of the wave function for the particle of interest. Without finding the proper interpolating operator contamination from other particle states can often occur. This does not cause serious difficulties when one deals with simple particles such as mesons or baryons, but the extraction of the correct mass can be a tremendous task when one is looking for more compound states. Therefore, a spectroscopic method that does not require knowledge of the wave function is desirable.

The question was first addressed in Ref. [1], where the explicit inversion of the fermion matrix on duplicated lattice configurations and the examination of the exponential decay rate of the lowest hadron state led to the expression

$$
a m_{\pi}=-\frac{1}{L_{t}} \cdot \max _{\left|\lambda_{k}\right|<1} \ln \left|\lambda_{k}\right|^{2}
$$

for the mass of the Goldstone pion on each configuration, where $a$ is the lattice spacing, $L_{t}$ is the number of lattice sites in the temporal direction and $\lambda_{k}$ are the eigenvalues of the reduced staggered fermion matrix (see Section 3). The aim is to find the relation between the hadron spectrum and these eigenvalues.

There has been many advances recently in the canonical approach to finite density QCD [2-5]. Based on the canonical formulation we make an attempt to clarify and extend the findings of Ref. [1] and give a method which in principle can be used to obtain the masses of different particles.

The paper is organized as follows. In Section 2 we summarize how canonical partition functions can be used to obtain the masses of particles. This is followed by a description of how canonical partition functions can be obtained on the lattice in Section 3. The way one can find the relevant eigenvalues is shown in section 4. In Section 5 we show how the $Z_{3}$ symmetry can be used to simplify our formulae. The case of baryons is explained in Section 6 while the case of mesons is discussed in Section 7 . Finally, after showing our numerical results in Section 8 we conclude in Section 9 , 


\section{Masses from canonical partition functions}

Let us consider the general case when we have $n_{s}$ different quark fields. Let $\hat{N}_{i}$ and $\mu_{i}$ denote the quark number operator and the quark number chemical potential corresponding to the $i$ th quark field, respectively. Then the grand canonical partition function at a given set of chemical potential values $\left(\mu_{1}, \mu_{2}, \ldots, \mu_{n_{s}}\right)$ and temperature $T$ is given by

$$
Z\left(\mu_{1}, \mu_{2}, \ldots, \mu_{n_{s}}, T\right)=\operatorname{Tr}\left[e^{-\left(\hat{H}-\mu_{1} \hat{N}_{1}-\mu_{2} \hat{N}_{2}-\ldots-\mu_{n_{s}} \hat{N}_{n_{s}}\right) / T}\right] .
$$

The canonical partition function corresponding to a given set of quark number values $N_{1}, \ldots, N_{n_{s}}$ can be obtained by taking the trace only over the subspace $N_{1}=N_{1}, \ldots, \hat{N}_{n_{s}}=N_{n_{s}}$.

$$
\begin{aligned}
Z_{N_{1}, \ldots, N_{n_{s}}}(T) & =\operatorname{Tr}\left[e^{-\hat{H} / T} \cdot \delta_{\hat{N}_{1}, N_{1}} \ldots \delta_{\hat{N}_{n_{s}}, N_{n_{s}}}\right] \\
& =\operatorname{Tr}\left[e^{-\hat{H} / T} \cdot \frac{1}{2 \pi} \int_{0}^{2 \pi} e^{i\left(\hat{N}_{1}-N_{1}\right) \theta_{1}} \mathrm{~d} \theta_{1} \ldots \frac{1}{2 \pi} \int_{0}^{2 \pi} e^{i\left(\hat{N}_{n_{s}}-N_{n_{s}}\right) \theta_{n_{s}}} \mathrm{~d} \theta_{n_{s}}\right] \\
& =\frac{1}{(2 \pi)^{n_{s}}} \int_{0}^{2 \pi} \mathrm{d} \theta_{1} e^{-i N_{1} \theta_{1}} \ldots \int_{0}^{2 \pi} \mathrm{d} \theta_{n_{s}} e^{-i N_{n_{s}} \theta_{n_{s}}} \operatorname{Tr}\left[e^{-\left(\hat{H}-i T \theta_{1} \hat{N}_{1}-\ldots-i T \theta_{n_{s}} \hat{N}_{n_{s}}\right) / T}\right] \\
& =\frac{1}{(2 \pi)^{n_{s}}} \int_{0}^{2 \pi} \mathrm{d} \theta_{1} e^{-i N_{1} \theta_{1}} \ldots \int_{0}^{2 \pi} \mathrm{d} \theta_{n_{s}} e^{-i N_{n_{s}} \theta_{n_{s}}} Z\left(i T \theta_{1}, \ldots, i T \theta_{n_{s}}, T\right)
\end{aligned}
$$

When one introduces imaginary chemical potentials [6], the different canonical partition functions become the coefficients in the Fourier expansion of the grand canonical partition function.

$$
\begin{gathered}
Z_{N_{1}, \ldots, N_{n_{s}}}(T)=\frac{1}{(2 \pi T)^{n_{s}}} \int_{0}^{2 \pi T} \mathrm{~d} \mu_{1} \ldots \int_{0}^{2 \pi T} \mathrm{~d} \mu_{n_{s}} e^{-i \mu_{1} N_{1} / T} \cdots e^{-i \mu_{n_{s}} N_{n_{s}} / T} Z\left(i \mu_{1}, \ldots, i \mu_{n_{s}}, T\right) \\
Z\left(i \mu_{1}, \ldots, i \mu_{n_{s}}, T\right)=\sum_{N_{1}=-\infty}^{\infty} \ldots \sum_{N_{n_{s}}=-\infty}^{\infty} Z_{N_{1}, \ldots, N_{n_{s}}}(T) e^{i \mu_{1} N_{1} / T} \cdots e^{i \mu_{n_{s}} N_{n_{s}} / T}
\end{gathered}
$$

When the aim is to find the energy of the lowest state in the sector corresponding to quark numbers $\left(N_{1}, \ldots, N_{n_{s}}\right)$ one has to examine the low temperature behavior of the free energy

$$
F_{N_{1}, \ldots, N_{n_{s}}}(T)=-T \ln Z_{N_{1}, \ldots, N_{n_{s}}}(T) .
$$

The canonical partition function can be written as

$$
Z_{N_{1}, \ldots, N_{n_{s}}}(T)=\sum_{k=0}^{\infty} n_{k}^{\left(N_{1}, \ldots, N_{n_{s}}\right)} e^{-E_{k}^{\left(N_{1}, \ldots, N_{n_{s}}\right)} / T}
$$

where $E_{k}^{\left(N_{1}, \ldots, N_{n_{s}}\right)}$ and $n_{k}^{\left(N_{1}, \ldots, N_{n_{s}}\right)}$ are the energy and the multiplicity of the $k$ th state in sector $\left(N_{1}, \ldots, N_{n_{s}}\right)$, respectively. In sector $(0, \ldots, 0)$ the lowest state is the vacuum, which is assumed to be non-degenerate.

$$
Z_{0, \ldots, 0}(T)=e^{-E_{0}^{(0, \ldots, 0)} / T}+\sum_{k=1}^{\infty} n_{k}^{(0, \ldots, 0)} e^{-E_{k}^{(0, \ldots, 0)} / T}
$$

Then the difference of the free energies of sector $\left(N_{1}, \ldots, N_{n_{s}}\right)$ and the zero quark number sector can be rewritten as

$$
\begin{aligned}
F_{N_{1}, \ldots, N_{n_{s}}}(T)-F_{0, \ldots, 0}(T)=E_{0}^{\left(N_{1}, \ldots, N_{n_{s}}\right)}-E_{0}^{(0, \ldots, 0)}-T \ln n_{0}^{\left(N_{1}, \ldots, N_{n_{s}}\right)} \\
-T \ln \left[\frac{1+\sum_{k=1}^{\infty} \frac{n_{k}^{\left(N_{1}, \ldots, N_{n_{s}}\right)}}{n_{0}^{\left(N_{1}, \ldots, N_{n_{s}}\right)}} e^{-\left(E_{k}^{\left(N_{1}, \ldots, N_{n_{s}}\right)}-E_{0}^{\left(N_{1}, \ldots, N_{n_{s}}\right)}\right) / T}}{1+\sum_{k=1}^{\infty} n_{k}^{(0, \ldots, 0)} e^{-\left(E_{k}^{(0, \ldots, 0)}-E_{0}^{(0, \ldots, 0)}\right) / T}}\right] .
\end{aligned}
$$


The mass of the lowest state in sector $\left(N_{1}, \ldots, N_{n_{s}}\right)$ is the difference of the energy of the ground state in this sector and the energy of the vacuum state,

$$
m_{0}^{\left(N_{1}, \ldots, N_{n_{s}}\right)}=E_{0}^{\left(N_{1}, \ldots, N_{n_{s}}\right)}-E_{0}^{(0, \ldots, 0)} .
$$

If the temperature is much smaller than the energy differences that appear in the exponentials in equation (9) then the last term on the r.h.s. of (9) is negligible compared to the other terms. In this region the difference of the free energies follows a linear behaviour,

$$
F_{N_{1}, \ldots, N_{n_{s}}}(T)-F_{0, \ldots, 0}(T) \approx m_{0}^{\left(N_{1}, \ldots, N_{n_{s}}\right)}-T \ln n_{0}^{\left(N_{1}, \ldots, N_{n_{s}}\right)},
$$

where the slope of the linear behaviour depends only on the multiplicity of the ground state. Therefore, the mass of the lightest particle carrying quantum numbers $\left(N_{1}, \ldots, N_{n_{s}}\right)$ and its multiplicity can be obtained by a linear extrapolation to the $T=0$ limit.

$$
m_{0}^{\left(N_{1}, \ldots, N_{n_{s}}\right)}=\lim _{T \rightarrow 0}\left[F_{N_{1}, \ldots, N_{n_{s}}}(T)-F_{0, \ldots, 0}(T)\right]
$$

\section{Canonical partition functions on the lattice}

The temperature on the lattice is given by $T=1 / a L_{t}$, where $L_{t}$ is the number of sites in the temporal direction and $a$ is the lattice spacing. Let $\hat{\mu}_{i}=\mu_{i} a$ denote the chemical potentials in lattice units. In order to introduce these chemical potentials on the lattice the forward time-like links have to be multiplied by $e^{i \hat{\mu}_{i}}$ and the backward time-like links by $e^{-i \hat{\mu}_{i}}$ in the fermion determinant of quarks of type $i$ [7]. Then the grand canonical partition function using staggered lattice fermions can be written as

$$
Z\left(i \hat{\mu}_{1}, \ldots, i \hat{\mu}_{n_{s}}\right)=\int[\mathrm{d} U] e^{-S_{g}[U]} \prod_{i=1}^{n_{s}} \operatorname{det} M\left(m_{i}, i \hat{\mu}_{i}, U\right)^{n_{i} / 4},
$$

where $m_{i}$ denotes the bare mass and $n_{i}$ denotes the number of tastes of the $i$ th staggered quark field. The functional integral is taken over all possible gauge configurations $U$ and $S_{g}[U]$ denotes the pure gauge part of the action.

The partition function can be rewritten in the form

$$
Z\left(i \hat{\mu}_{1}, \ldots, i \hat{\mu}_{n_{s}}\right)=\int[\mathrm{d} U] e^{-S_{g}[U]} \prod_{i=1}^{n_{s}} \operatorname{det} M\left(m_{i}, 0, U\right)^{n_{i} / 4} \times \prod_{i=1}^{n_{s}}\left(\frac{\operatorname{det} M\left(m_{i}, i \hat{\mu}_{i}, U\right)}{\operatorname{det} M\left(m_{i}, 0, U\right)}\right)^{n_{i} / 4} .
$$

The ratios of the determinants can be treated as observables while the functional integral can be taken using the measure at $\hat{\mu}_{i}=0$. Then the partition function becomes the expectation value of the determinant ratios taken over the ensemble generated at zero chemical potentials,

$$
Z\left(i \hat{\mu}_{1}, \ldots, i \hat{\mu}_{n_{s}}\right)=Z \cdot\left\langle\prod_{i=1}^{n_{s}}\left(\frac{\operatorname{det} M\left(m_{i}, i \hat{\mu}_{i}, U\right)}{\operatorname{det} M\left(m_{i}, 0, U\right)}\right)^{n_{i} / 4}\right\rangle,
$$

where $Z$ denotes the zero chemical potential value of the partition function [8].

Therefore, the canonical partition functions are obtained by taking the expectation values of the Fourier components of the determinant ratios.

$$
Z_{N_{1}, \ldots, N_{n_{s}}}=Z \cdot\left\langle\prod_{i=1}^{n_{s}} \frac{L_{t}}{2 \pi} \int_{0}^{\frac{2 \pi}{L_{t}}} \mathrm{~d} \hat{\mu}_{i} e^{-i \hat{\mu}_{i} N_{i} L_{t}}\left(\frac{\operatorname{det} M\left(m_{i}, i \hat{\mu}_{i}, U\right)}{\operatorname{det} M\left(m_{i}, 0, U\right)}\right)^{n_{i} / 4}\right\rangle
$$

In order to perform the assigned Fourier transformations we need the analytic $\hat{\mu}-\operatorname{dependence~of~} \operatorname{det} M(i \hat{\mu})$. In temporal gauge, the fermion matrix can be written as

$$
M(i \hat{\mu})=\left(\begin{array}{cccccc}
B_{0} & e^{i \hat{\mu}} & 0 & \ldots & 0 & U e^{-i \hat{\mu}} \\
-e^{-i \hat{\mu}} & B_{1} & e^{i \hat{\mu}} & \ldots & 0 & 0 \\
0 & -e^{-i \hat{\mu}} & B_{2} & \ldots & 0 & 0 \\
\vdots & \vdots & \vdots & \ddots & \vdots & \vdots \\
0 & 0 & 0 & \ldots & B_{L_{t}-2} & e^{i \hat{\mu}} \\
-U^{\dagger} e^{i \hat{\mu}} & 0 & 0 & \ldots & -e^{-i \hat{\mu}} & B_{L_{t}-1}
\end{array}\right)
$$


where $\mathrm{U}$ denotes the remaining time direction links on the last timeslice (including the correct staggered phases) and $B_{k}$ is the spacelike staggered fermion matrix on timeslice $k$. In matrix (17) each block is a $3 V \times 3 V$ matrix, where $V=L_{s}^{3}$ and $L_{s}$ is the spatial size of the lattice. After performing $L_{t}-2$ steps of Gaussian elimination, the determinant of (17) can be written as

$$
\operatorname{det} M(i \hat{\mu})=e^{3 V L_{t} i \hat{\mu}} \operatorname{det}\left(S-e^{-i \hat{\mu} L_{t}}\right) \text {, }
$$

where

$$
S=\left(\begin{array}{cc}
0 & 1 \\
1 & B_{L_{t}-1}
\end{array}\right)\left(\begin{array}{cc}
0 & 1 \\
1 & B_{L_{t}-2}
\end{array}\right) \cdots\left(\begin{array}{cc}
0 & 1 \\
1 & B_{0}
\end{array}\right)\left(\begin{array}{cc}
U & 0 \\
0 & U
\end{array}\right)
$$

is the $6 \mathrm{~V} \times 6 \mathrm{~V}$ sized reduced fermion matrix [9]. Let $\lambda_{k}$ denote the eigenvalues of $S$. Then (18) can be written as

$$
\operatorname{det} M(i \hat{\mu})=e^{3 V L_{t} i \hat{\mu}} \prod_{k=1}^{6 V}\left(\lambda_{k}-e^{-i \hat{\mu} L_{t}}\right)
$$

and thus,

$$
\frac{\operatorname{det} M(i \hat{\mu})}{\operatorname{det} M(0)}=e^{3 V L_{t} i \hat{\mu}} \prod_{k=1}^{6 V} \frac{\lambda_{k}-e^{-i \hat{\mu} L_{t}}}{\lambda_{k}-1} .
$$

The eigenvalues of matrix $S$ have a symmetry, according to which whenever $\lambda$ is an eigenvalue of $S$ then $1 / \lambda^{*}$ is also an eigenvalue of $S[9]$. Therefore, each eigenvalue whose absolute value is greater than 1 has a pair with an absolute value smaller than 1 , and vice versa. (We will not deal with the case when at least one of the eigenvalues lie on the unit circle because these gauge configurations constitute a zero measure set.) Then (21) can be written as

$$
\frac{\operatorname{det} M(i \hat{\mu})}{\operatorname{det} M(0)}=e^{3 V L_{t} i \hat{\mu}} \prod_{k=1}^{3 V} \frac{\lambda_{k}-e^{-i \hat{\mu} L_{t}}}{\lambda_{k}-1} \frac{\frac{1}{\lambda_{k}^{*}}-e^{-i \hat{\mu} L_{t}}}{\frac{1}{\lambda_{k}^{*}}-1}=\prod_{k=1}^{3 V}\left|\frac{1-\lambda_{k} e^{i \hat{\mu} L_{t}}}{1-\lambda_{k}}\right|^{2}
$$

where the product is taken over only the eigenvalues lying inside the unit circle. From now on when the limits of a sum or product taken over the eigenvalues of $S$ are from 1 to $3 \mathrm{~V}$, then the sum or product is meant to be taken over only the "small" eigenvalues, that is, the eigenvalues with absolute value smaller than 1 .

When the temperature is low $\left(T \ll T_{c}\right)$ a gap appears between the "small" and "large" eigenvalues of $S$ (see Figure 11). This makes a Taylor expansion of (22) in the small eigenvalues possible. As the temperature decreases the small eigenvalues become exponentially smaller, increasing the validity of the series expansion. Including the rational exponent for the number of tastes $n_{t}$ a first order expansion gives

$$
\begin{aligned}
\left(\frac{\operatorname{det} M(i \hat{\mu})}{\operatorname{det} M(0)}\right)^{n_{t} / 4} & =\left(\prod_{k=1}^{3 V}\left|\frac{1-\lambda_{k} e^{i \hat{\mu} L_{t}}}{1-\lambda_{k}}\right|^{2}\right)^{n_{t} / 4} \\
& \approx\left[1+\frac{n_{t}}{4} \sum_{k=1}^{3 V} \lambda_{k}+\frac{n_{t}}{4} \sum_{k=1}^{3 V} \lambda_{k}^{*}\right]+e^{i \hat{\mu} L_{t}}\left[-\frac{n_{t}}{4} \sum_{k=1}^{3 V} \lambda_{k}\right]+e^{-i \hat{\mu} L_{t}}\left[-\frac{n_{t}}{4} \sum_{k=1}^{3 V} \lambda_{k}^{*}\right]
\end{aligned}
$$

By performing an $n$th order Taylor expansion we explicitly obtain all the Fourier coefficients up to $n$th order.

This way the assigned Fourier transformations in equation (16) can be performed easily configuration by configuration by simply choosing the coefficients of the corresponding exponential terms. The order of the leading order term for sector $\left(N_{1}, \ldots, N_{n_{s}}\right)$ is $\left|N_{1}\right|+\cdots+\left|N_{n_{s}}\right|$. When all the quark fields have 4 tastes $\left(n_{i}=4\right)$ the leading order term for sector $\left(N_{1}, \ldots, N_{n_{s}}\right)$ can be written as

$$
Z_{N_{1}, \ldots, N_{n_{s}}} \stackrel{\mathrm{LO}}{=} Z \cdot\left\langle\prod_{i=1}^{n_{s}}\left[(-1)^{\left|N_{i}\right|} \sum_{1 \leq k_{1}^{(i)}<\cdots<k_{\left|N_{i}\right|}^{(i)} \leq 3 V}\left(\lambda_{k_{1}^{(i)}}^{(i)} \cdots \lambda_{k_{\left|N_{i}\right|}^{(i)}}^{(i)}\right)^{*\left(\operatorname{sgn} N_{i}\right)}\right]\right\rangle
$$

where $*\left(\operatorname{sgn} N_{i}\right)$ in the exponent means that there is a complex conjugation if $N_{i}$ is negative. $\lambda_{k}^{(i)}$ stands for the $k$ th eigenvalue of the reduced matrix $S^{(i)}$ obtained from the fermion matrix of the $i$ th quark field. 


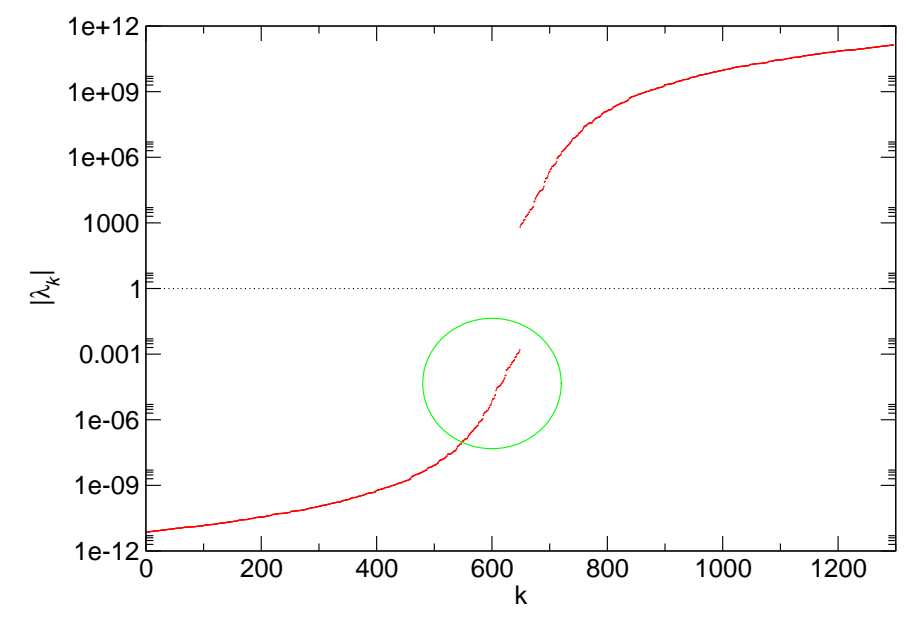

Figure 1: The absolute values of the eigenvalues of the reduced fermion matrix $S$ on a typical $6^{3} \times 24$ lattice configuration at a temperature of $T \approx 25 \mathrm{MeV}$. At this temperature there is already a noticeable gap between the eigenvalues lying inside the unit circle and the ones lying outside. The eigenvalues that are relevant for calculating the canonical partition functions are circled.

The leading order term in case of arbitrary number of tastes $n_{i}$ can be obtained from (24) as follows. The formula within the expectation value signs can be written as a homogeneous polynomial of the eigenvalues of degree $\left|N_{1}\right|+\cdots+\left|N_{n_{s}}\right|$ using the expressions

$$
\begin{gathered}
\sum_{k=1}^{3 V}\left(\lambda_{k}^{(i)}\right)^{j} \quad j=1, \ldots, N_{i} \quad \text { if } N_{i} \text { is positive, and } \\
\sum_{k=1}^{3 V}\left(\lambda_{k}^{(i))^{*}}\right)^{j} \quad j=1, \ldots,-N_{i} \quad \text { if } N_{i} \text { is negative. }
\end{gathered}
$$

The leading order term in the general case is obtained by replacing the expressions

$$
\sum_{k=1}^{3 V}\left(\lambda_{k}^{(i)}\right)^{j} \quad \text { with } \frac{n_{i}}{4} \sum_{k=1}^{3 V}\left(\lambda_{k}^{(i)}\right)^{j} \quad \text { and } \quad \sum_{k=1}^{3 V}\left(\lambda_{k}^{(i)^{*}}\right)^{j} \quad \text { with } \quad \frac{n_{i}}{4} \sum_{k=1}^{3 V}\left(\lambda_{k}^{(i) *}\right)^{j}
$$

in the above polynomial.

\section{Obtaining the relevant eigenvalues}

In order to calculate the canonical partition functions using the description given in (24), (25) and (26) we do not need all of the $3 V$ small eigenvalues. At lower temperatures $\left(L_{t} \geq 50-100\right)$ the small eigenvalues alone span a range of $20-40$ orders of magnitude. Therefore, the relevant eigenvalues that contribute significantly to the sums in (24) -(26) are the largest few of the small eigenvalues (see Figure11). Since the condition number of matrix $S$ at low temperatures can be in the range of $O\left(10^{60}-10^{100}\right)$ and these relevant eigenvalues are in the middle of the spectrum, finding these eigenvalues seems practically impossible.

Nevertheless, the matrix $S$ has some symmetry properties that make it possible. The spacelike staggered fermion matrices $B_{k}$, which appear in (17) and (19), obey a $\gamma_{5}$-hermiticity

$$
\gamma_{5} B_{k}=B_{k}^{\dagger} \gamma_{5},
$$

where

$$
\left(\gamma_{5}\right)_{x y}=\delta_{x y} \cdot(-1)^{\sum_{\mu} x_{\mu}} .
$$




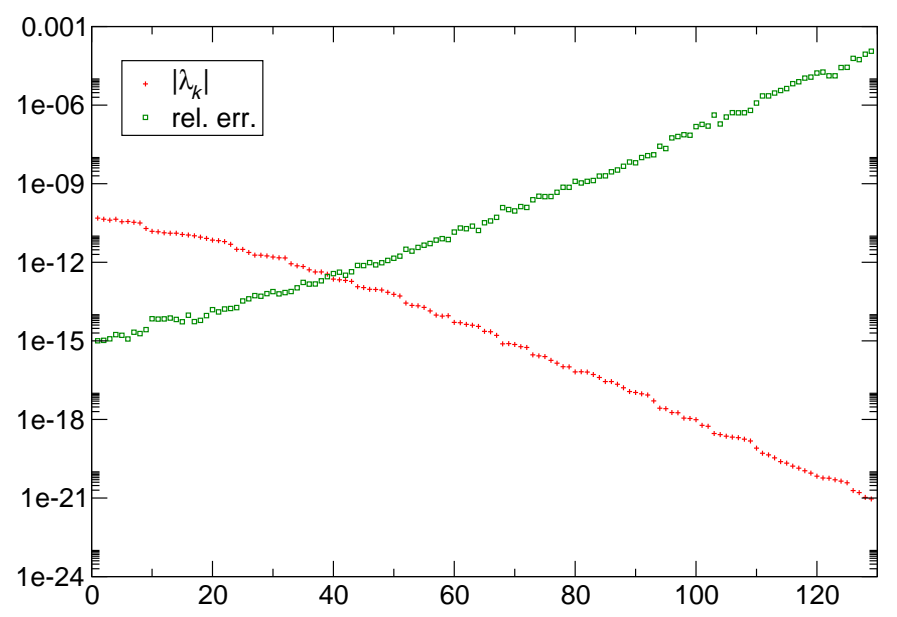

Figure 2: The absolute values of the relevant eigenvalues found by the double precision version of ARPACK (red crosses) and their relative errors (green squares) on a typical lattice of size $6^{3} \times 100$.

Therefore, the inverse of $S$ can be obtained as

$$
S^{-1}=(-1)^{L_{t}+1}\left(\begin{array}{cc}
0 & -\gamma_{5} \\
\gamma_{5} & 0
\end{array}\right) S^{\dagger}\left(\begin{array}{cc}
0 & -\gamma_{5} \\
\gamma_{5} & 0
\end{array}\right) .
$$

As a consequence, once we have the matrix $S$ both $S+S^{-1}$ and $S-S^{-1}$ can be easily constructed. Then by inverting these two one can arrive at

$$
Q=\frac{1}{2}\left[\left(S+S^{-1}\right)^{-1}-\left(S-S^{-1}\right)^{-1}\right] .
$$

The order of magnitude of the condition number of $S+S^{-1}$ and $S-S^{-1}$ is less than half of that of $S$. Therefore, much less numerical precision is sufficient for their inversion.

If $\lambda_{k}$ is an eigenvalue of $S$ then $\lambda_{k} /\left(1-\lambda_{k}^{4}\right)$ is an eigenvalue of $Q$. If $\lambda_{k}$ is a small eigenvalue, then $\left|\lambda_{k}^{4}\right| \lll 1$. In this case using $\lambda_{k} /\left(1-\lambda_{k}^{4}\right)$ for the calculations instead of $\lambda_{k}$ does not make any difference. If $\lambda_{k}$ is a large eigenvalue of $S$, then $\lambda_{k} /\left(1-\lambda_{k}^{4}\right) \approx-1 / \lambda_{k}^{3}$, which is negligible compared to the relevant small eigenvalues. That is, the relevant eigenvalues of $S$ become the largest eigenvalues of $Q$.

The procedure for finding the relevant eigenvalues was as follows. After fixing the temporal gauge the matrices $B_{k}$ were built. Then from (19) and (29) the matrices $S$ and $S^{-1}$ were constructed. Since $S$ and $S^{-1}$ are very badly conditioned, their construction as well as working with them requires high precision. For these and the latter calculations the GNU multiple precision arithmetic library (GNU MP) was used. Then after inverting $S+S^{-1}$ and $S-S^{-1}$ the largest several eigenvalues of $Q$ (the relevant ones) were obtained using the double precision version of ARPACK. This last step may sound doubious but in fact the double ARPACK was found to be able to reliably find the eigenvalues that were not more than 10 orders of magnitude smaller than the largest one (see Figure 2).

\section{Consequences of the $Z_{3}$ symmetry}

\subsection{Consequences for $Z_{N_{1}, \ldots, N_{n_{s}}}$}

From (22) it can be seen that the quantity within the expectation value signs in equation (15) is periodic in each $\hat{\mu}_{i}$ with a periodicity of $2 \pi / L_{t}$ configuration by configuration. Therefore, the lattice grand canonical partition function (13) is also periodic with $2 \pi / L_{t}$ in each $\hat{\mu}_{i}$.

Performing a $Z_{3}$ transformation, that is, multiplying all the time-like links on the last timeslice of an $S U(3)$ configuration $U$ by $\varepsilon_{j}\left(\varepsilon_{j}=e^{2 \pi i \cdot j / 3}, j=0,1,2\right)$ gives another $S U(3)$ configuration denoted by $U^{\varepsilon_{j}}$. 
Then the partition function can be written as

$$
Z\left(i \hat{\mu}_{1}, \ldots, i \hat{\mu}_{n_{s}}\right)=\frac{1}{3} \sum_{j=0}^{2} \int\left[\mathrm{d} U^{\varepsilon_{j}}\right] e^{-S_{g}\left[U^{\varepsilon_{j}}\right]} \prod_{i=1}^{n_{s}} \operatorname{det} M\left(m_{i}, i \hat{\mu}_{i}, U^{\varepsilon_{j}}\right)^{n_{i} / 4}
$$

The functional measure and the gauge action are both symmetric with respect to $Z_{3}$ transformations [10]. Thus,

$$
\begin{aligned}
Z\left(i \hat{\mu}_{1}, \ldots, i \hat{\mu}_{n_{s}}\right) & =\int[\mathrm{d} U] e^{-S_{g}[U]} \frac{1}{3} \sum_{j=0}^{2} \prod_{i=1}^{n_{s}} \operatorname{det} M\left(m_{i}, i \hat{\mu}_{i}, U^{\varepsilon_{j}}\right)^{n_{i} / 4} \\
& =\int[\mathrm{d} U] e^{-S_{g}[U]} \prod_{i=1}^{n_{s}} \operatorname{det} M\left(m_{i}, 0, U\right)^{n_{i} / 4} \times \frac{1}{3} \sum_{j=0}^{2} \prod_{i=1}^{n_{s}}\left(\frac{\operatorname{det} M\left(m_{i}, i \hat{\mu}_{i}, U^{\varepsilon_{j}}\right)}{\operatorname{det} M\left(m_{i}, 0, U\right)}\right)^{n_{i} / 4} \\
& =Z \cdot\left\langle\frac{1}{3} \sum_{j=0}^{2} \prod_{i=1}^{n_{s}}\left(\frac{\operatorname{det} M\left(m_{i}, i \hat{\mu}_{i}, U^{\varepsilon_{j}}\right)}{\operatorname{det} M\left(m_{i}, 0, U\right)}\right)^{n_{i} / 4}\right\rangle .
\end{aligned}
$$

Since the $U \rightarrow U^{\varepsilon_{j}}$ transformation can be applied in eq. (18) by simply multiplying $S$ by $\varepsilon_{j}$, the ratios of the determinants in (32) can be rewritten as

$$
\begin{aligned}
\frac{\operatorname{det} M\left(m_{i}, i \hat{\mu}_{i}, U^{\varepsilon_{j}}\right)}{\operatorname{det} M\left(m_{i}, 0, U\right)} & =\prod_{k=1}^{3 V}\left|\frac{1-\lambda_{k}^{(i)} \varepsilon_{j} e^{i \hat{\mu}_{i} L_{t}}}{1-\lambda_{k}^{(i)}}\right|^{2}=\prod_{k=1}^{3 V}\left|\frac{1-\lambda_{k}^{(i)} e^{i \hat{\mu}_{i} L_{t}+i \frac{2 \pi}{3} j}}{1-\lambda_{k}^{(i)}}\right|^{2} \\
& =\frac{\operatorname{det} M\left(m_{i}, i \hat{\mu}_{i}+i \frac{2 \pi}{3 L_{t}}, U\right)}{\operatorname{det} M\left(m_{i}, 0, U\right)}
\end{aligned}
$$

Combining (33) with (32) we obtain

$$
\begin{array}{r}
Z\left(i \hat{\mu}_{1}, \ldots, i \hat{\mu}_{n_{s}}\right)=\frac{1}{3}\left[Z\left(i \hat{\mu}_{1}, \ldots, i \hat{\mu}_{n_{s}}\right)+Z\left(i \hat{\mu}_{1}+i \frac{2 \pi}{3 L_{t}}, \ldots, i \hat{\mu}_{n_{s}}+i \frac{2 \pi}{3 L_{t}}\right)\right. \\
\left.+Z\left(i \hat{\mu}_{1}+i \frac{4 \pi}{3 L_{t}}, \ldots, i \hat{\mu}_{n_{s}}+i \frac{4 \pi}{3 L_{t}}\right)\right]
\end{array}
$$

which means that the grand canonical partition function has an extra periodicity: if $2 \pi i / 3 L_{t}$ is added to all the chemical potentials then the value of the partition function remains unchanged [6].

$$
Z\left(i \hat{\mu}_{1}, \ldots, i \hat{\mu}_{n_{s}}\right)=Z\left(i \hat{\mu}_{1}+i \frac{2 \pi}{3 L_{t}}, \ldots, i \hat{\mu}_{n_{s}}+i \frac{2 \pi}{3 L_{t}}\right)
$$

Therefore, the canonical partition functions $Z_{N_{1}, \ldots, N_{n_{s}}}$ where the total number of quarks $N_{1}+\cdots+N_{n_{s}}$ is not divisible by 3 are zero [4].

Taking this into account the expectation value of the first order expansion in (23) gives

$$
\left\langle\left(\frac{\operatorname{det} M(i \hat{\mu})}{\operatorname{det} M(0)}\right)^{n_{t} / 4}\right\rangle \approx\left\langle 1+\frac{n_{t}}{4} \sum_{k=1}^{3 V} \lambda_{k}+\frac{n_{t}}{4} \sum_{k=1}^{3 V} \lambda_{k}^{*}\right\rangle
$$

\subsection{Application on a term by term basis}

Let $A[U]$ be a gauge invariant quantity (a gauge invariant function of the gauge configuration $U$ ). Then the expectation value of $A[U]$ is

$$
\langle A[U]\rangle=\frac{1}{Z} \int[\mathrm{d} U] e^{-S_{g}[U]} \operatorname{det} M\left(m_{1}, 0, U\right)^{n_{1} / 4} \cdots \operatorname{det} M\left(m_{n_{s}}, 0, U\right)^{n_{n_{s}} / 4} \cdot A[U] .
$$


Using the $Z_{3}$ invariance of the gauge action and the integration measure one can rewrite (37) as

$$
\langle A[U]\rangle=\frac{1}{Z} \int[\mathrm{d} U] e^{-S_{g}[U]} \prod_{i=1}^{n_{s}} \operatorname{det} M\left(m_{i}, 0, U\right)^{n_{i} / 4} \times \frac{1}{3} \sum_{j=0}^{2} A\left[U^{\varepsilon_{j}}\right] \cdot \prod_{i=1}^{n_{s}}\left(\frac{\operatorname{det} M\left(m_{i}, 0, U^{\varepsilon_{j}}\right)}{\operatorname{det} M\left(m_{i}, 0, U\right)}\right)^{n_{i} / 4},
$$

that is,

$$
\langle A[U]\rangle=\left\langle\frac{1}{3} \sum_{j=0}^{2} A\left[U^{\varepsilon_{j}}\right] \cdot \prod_{i=1}^{n_{s}}\left(\frac{\operatorname{det} M\left(m_{i}, 0, U^{\varepsilon_{j}}\right)}{\operatorname{det} M\left(m_{i}, 0, U\right)}\right)^{n_{i} / 4}\right\rangle .
$$

Using (33) an expansion similar to (23) can be applied to these determinant ratios.

All the quantities of the form of (25) are gauge independent, therefore, each term of the series expansion can individually be taken as $A[U]$. This way the $Z_{3}$ symmetric form of (39) can be applied to each term in the series expansion. As an example, if we have only one staggered field $\left(n_{s}=1\right)$ with number of tastes $n_{t}$ then the expectation value of the first order terms in eq. (23) up to leading order become

$$
\left\langle\sum_{k=1}^{3 V} \lambda_{k}\right\rangle \stackrel{\mathrm{LO}}{=}\left\langle\sum_{k=1}^{3 V} \lambda_{k}^{*}\right\rangle \stackrel{\mathrm{LO}}{=}\left\langle-\frac{n_{t}}{4}\left|\sum_{k=1}^{3 V} \lambda_{k}\right|^{2}\right\rangle
$$

Applying this technique term by term the series expansion of (23) and (36) up to third order becomes

$$
\begin{aligned}
\left\langle\left(\frac{\operatorname{det} M(i \hat{\mu})}{\operatorname{det} M(0)}\right)^{n_{t} / 4}\right\rangle & \approx\left\langle 1+\frac{n_{t}}{12} \sum_{k=1}^{3 V} \lambda_{k}^{3}-\frac{n_{t}^{2}}{32}\left(\sum_{k=1}^{3 V} \lambda_{k}\right)\left(\sum_{k=1}^{3 V} \lambda_{k}^{2}\right)+\frac{n_{t}^{3}}{384}\left(\sum_{k=1}^{3 V} \lambda_{k}\right)^{3}\right. \\
& \left.+\frac{n_{t}}{12} \sum_{k=1}^{3 V} \lambda_{k}^{* 3}-\frac{n_{t}^{2}}{32}\left(\sum_{k=1}^{3 V} \lambda_{k}^{*}\right)\left(\sum_{k=1}^{3 V} \lambda_{k}^{* 2}\right)+\frac{n_{t}^{3}}{384}\left(\sum_{k=1}^{3 V} \lambda_{k}^{*}\right)^{3}\right\rangle \\
+e^{3 i \hat{\mu} L_{t}} & \cdot\left\langle-\frac{n_{t}}{12} \sum_{k=1}^{3 V} \lambda_{k}^{3}+\frac{n_{t}^{2}}{32}\left(\sum_{k=1}^{3 V} \lambda_{k}\right)\left(\sum_{k=1}^{3 V} \lambda_{k}^{2}\right)-\frac{n_{t}^{3}}{384}\left(\sum_{k=1}^{3 V} \lambda_{k}\right)^{3}\right\rangle \\
& +e^{-3 i \hat{\mu} L_{t}} \cdot\left\langle-\frac{n_{t}}{12} \sum_{k=1}^{3 V} \lambda_{k}^{* 3}+\frac{n_{t}^{2}}{32}\left(\sum_{k=1}^{3 V} \lambda_{k}^{*}\right)\left(\sum_{k=1}^{3 V} \lambda_{k}^{* 2}\right)-\frac{n_{t}^{3}}{384}\left(\sum_{k=1}^{3 V} \lambda_{k}^{*}\right)^{3}\right\rangle
\end{aligned}
$$

In the third order expansion in (41) all the terms are already $Z_{3}$ invariant. If a term in the series expansion is $Z_{3}$ invariant then it does not change when the procedure of (39) is applied to it. When the procedure (39) is applied to a non $-Z_{3}$ invariant term, its expectation value becomes the expectation value of the sum of higher order terms. This procedure can be continued order by order and as a result, all the remaining terms in all the quark number sectors of the series expansion of $Z\left(i \hat{\mu}_{1}, \ldots, i \hat{\mu}_{n_{s}}\right)$ become $Z_{3}$ invariant.

\section{Application to baryons}

In principle the method described in Sections 2 and 3 can be used to measure the mass of the lowest state in any quark number sector. For example, one can think of the di-baryon (deuteron), or the bound states of even more baryons. However, in most cases technical difficulties occur. Let us examine the case when one tries to measure the mass of a baryon, for example the proton. For that we use two staggered quark fields, one for the $u$ quark with $n_{u}$ tastes and one for the $d$ quark with $n_{d}$ tastes. (We omitted the third light quark, the $s$ quark, the inclusion of which in our case does not change the picture significantly.)

The proton is believed to be the lowest state in the $N_{u}=2, N_{d}=1$ channel, therefore, according to (11) we need to examine the low temperature behaviour of

$$
F_{N_{u}=2, N_{d}=1}(T)-F_{N_{u}=0, N_{d}=0}(T)=-T \ln \left(\frac{Z_{2,1}(T)}{Z_{0,0}(T)}\right) .
$$


According to (24)-(26) the relevant canonical partition functions to leading order are

$$
\begin{aligned}
& Z_{2,1} \stackrel{\mathrm{LO}}{=} Z \cdot\left\langle\frac{n_{u} n_{d}}{32}\left(\sum_{k=1}^{3 V} \lambda_{k}^{(u)^{2}}\right)\left(\sum_{k=1}^{3 V} \lambda_{k}^{(d)}\right)-\frac{n_{u}^{2} n_{d}}{128}\left(\sum_{k=1}^{3 V} \lambda_{k}^{(u)}\right)^{2}\left(\sum_{k=1}^{3 V} \lambda_{k}^{(d)}\right)\right\rangle, \\
& Z_{0,0} \stackrel{\mathrm{LO}}{=} Z \cdot\langle 1\rangle,
\end{aligned}
$$

therefore, for the proton mass one obtains

$$
a m_{p}=\lim _{L_{t} \rightarrow \infty}-\frac{1}{L_{t}} \ln \left\langle\frac{n_{u} n_{d}}{32}\left(\sum_{k=1}^{3 V} \lambda_{k}^{(u)^{2}}\right)\left(\sum_{k=1}^{3 V} \lambda_{k}^{(d)}\right)-\frac{n_{u}^{2} n_{d}}{128}\left(\sum_{k=1}^{3 V} \lambda_{k}^{(u)}\right)^{2}\left(\sum_{k=1}^{3 V} \lambda_{k}^{(d)}\right)\right\rangle .
$$

As the temperature decreases ( $L_{t}$ increases) the eigenvalues become smaller and smaller, and only the leading order term matters in the limit.

The formulae for the masses of the 2-baryon, 3-baryon, etc. channels can be obtained similarly. These can in principle be used to measure the bonding energy of several-baryon states.

The description seems simple, but there is one difficulty. The expression of which the expectation value is taken in equation (44) can be any complex number, whose real part can be both positive and negative. Its expectation value is much smaller than its value at a typical gauge configuration. On a $6^{3} \times 24$ staggered lattice with $a \approx 0.33 \mathrm{fm}, m_{\pi} \approx 330 \mathrm{MeV}$ and $T \approx 25 \mathrm{MeV}$ this value at a typical gauge configuration is of $O\left(10^{-10}\right)$ while the expected order of magnitude of the expectation value is $O\left(10^{-20}\right)$. That means that the number of configurations needed for a correct result would be of $O\left(10^{20}\right)$, which is prohibitive. The problem becomes even more severe when one decreases the temperature in order to get closer to the $T \rightarrow 0$ limit.

\section{Application to mesons}

When $n_{d}=n_{u}=n_{t} / 2, m_{d}=m_{u}$ and we are looking at one of the $N_{d}=-N_{u}$ sectors this sign problem does not arise. These sectors can be labelled with one parameter, the third component of the isospin $I_{3}=\left(N_{u}-N_{d}\right) / 2$. Since $\lambda_{k}^{(u)}=\lambda_{k}^{(d)}$ for all $k$, we will write $\lambda_{k}$ only.

The lowest state in the $I_{3}=1$ sector is expected to be the Goldstone pion. Its partition function can be written as the expectation value

$$
Z_{I_{3}=1} \stackrel{\mathrm{LO}}{=} Z_{N_{u}=1, N_{d}=-1} \stackrel{\mathrm{LO}}{=} Z \cdot\left\langle\frac{n_{t}^{2}}{64}\left|\sum_{k=1}^{3 V} \lambda_{k}\right|^{2}\right\rangle,
$$

which is a manifestly positive polynomial of the eigenvalues. Therefore, it can be easily evaluated, and by taking the zero temperature limit

$$
a m_{I_{3}=1, \pi}=\lim _{L_{t} \rightarrow \infty}-\frac{1}{L_{t}} \ln \left\langle\frac{n_{t}^{2}}{64}\left|\sum_{k=1}^{3 V} \lambda_{k}\right|^{2}\right\rangle
$$

one directly obtains the mass of the lowest state in the $I_{3}=1$ channel.

The formulae for the energies of the lowest state in higher $I_{3}$ channels can be obtained similarly. These can be used to investigate pion-pion scattering and several-pion states.

The result for the pion mass given in equation (46) obtained using purely thermodynamic considerations can be compared to formula (11). If $L_{t}$ is large, then after taking the logarithm the factor $n_{t}^{2} / 64$ gives a negligible contribution compared to that of the sum. If the temporal extension is large even compared to the spatial volume, then the sum in (46) is dominated by the largest of the small eigenvalues. In this case equations (46) and (11) evaluated on a single configuration yield approximately the same results. However, while Ref. [1] only states that relation (11) holds configuration by configuration and does not mention how to obtain results over an ensemble of configurations, equation (46) describes a method for taking the ensemble average. 


\begin{tabular}{c|ccc}
\hline \hline $1 / a T$ & \multicolumn{3}{|c}{ Number of configurations } \\
& $n_{t}=2$ & $n_{t}=4$ & $n_{t}=8$ \\
\hline 50 & 331 & 322 & - \\
100 & 1196 & 935 & 701 \\
200 & 323 & 605 & 467 \\
300 & 168 & 255 & - \\
\hline \hline
\end{tabular}

Table 1: The number of configurations used for dynamical staggered calculations with a spatial volume of $6^{3}$.

\begin{tabular}{c|ccc}
\hline \hline $1 / a T$ & \multicolumn{3}{|c}{$a F_{I_{3}=1}-a F_{I_{3}=0}$} \\
& $n_{t}=2$ & $n_{t}=4$ & $n_{t}=8$ \\
\hline 50 & $0.5344(12)$ & $0.4971(12)$ & - \\
100 & $0.5066(2)$ & $0.4826(4)$ & $0.4639(4)$ \\
200 & $0.4931(2)$ & $0.4760(1)$ & $0.4641(3)$ \\
300 & $0.4876(3)$ & $0.4730(3)$ & - \\
\hline$\rightarrow \infty$ & $0.4787(3)$ & $0.4688(3)$ & $0.4643(7)$ \\
\hline$a m_{\pi, \mathrm{sp}}$ & $0.47864(3)$ & $0.46903(4)$ & $0.46426(3)$ \\
\hline \hline
\end{tabular}

Table 2: The differences of the free energies, their $T \rightarrow 0$ extrapolated values and the spectroscopic pion masses on dynamical staggered configurations with a spatial volume of $6^{3}$.

\section{Results}

\subsection{Dynamical staggered fermions}

We performed calculations using dynamical staggered configurations to measure the masses in the first isospin channel as described in Section 7 . We used the Wilson plaquette action for the gauge fields and unimproved staggered fermion action. In order to be able to check whether the root taking of the fermion determinant changes the results significantly, calculations were done using rooted staggered fermions with $n_{t}=2\left(n_{u}=n_{d}=1\right)$ and $n_{t}=4\left(n_{u}=n_{d}=2\right)$ as well as unrooted fermions with $n_{t}=8\left(n_{u}=n_{d}=4\right)$.

For the $n_{t}=2$ runs the gauge coupling was $\beta=4.8$. The lattice spacing was $a=0.41 \mathrm{fm}$, measured from the string tension $\sigma$ using the value of $\sqrt{\sigma}=465 \mathrm{MeV}$ [11]. For the $n_{t}=4$ case $\beta=4.3$ and $a=0.42 \mathrm{fm}$ and for the $n_{t}=8$ case $\beta=3.8$ and $a=0.44 \mathrm{fm}$. In all three cases the bare quark mass was $a m_{q}=0.04$ and the spatial extension of the lattice was $L_{s}=6$. In the two rooted case we used temporal lattice extensions of $L_{t}=50,100,200,300$ while in the unrooted case only $L_{t}=100,200$ was used. Table 1 contains the number of configurations for each setup.

Using equation (45) the difference of the free energies $a F_{I_{3}=1}-a F_{I_{3}=0}$ were measured on each set of configurations. These are listed in Table 2. According to equation (11) the mass of the ground state in the $I_{3}=1$ channel can be obtained using a linear extrapolation to $T=0$. For comparison we measured the pion mass in all cases using the ordinary spectroscopic method, which will be denoted by $m_{\pi, \mathrm{sp}}$. The measured free energy values, the linear fits and the comparisons to the spectroscopic pion masses can be seen in Figure 3.

As can be seen from Figure 3 the mass of the ground state in the $I_{3}=1$ sector agrees with the spectroscopic pion mass within errorbars for both rooted and unrooted staggered fermions.

\subsection{Quenched case}

Equation (46) can be rewritten as

$$
a m_{I_{3}=1, \pi}=\lim _{L_{t} \rightarrow \infty}\left[-\frac{1}{L_{t}} \ln \left(\frac{n_{t}^{2}}{64}\right)-\frac{1}{L_{t}} \ln \left\langle\left|\sum_{k=1}^{3 V} \lambda_{k}\right|^{2}\right\rangle\right]=\lim _{L_{t} \rightarrow \infty}-\frac{1}{L_{t}} \ln \left\langle\left|\sum_{k=1}^{3 V} \lambda_{k}\right|^{2}\right\rangle .
$$




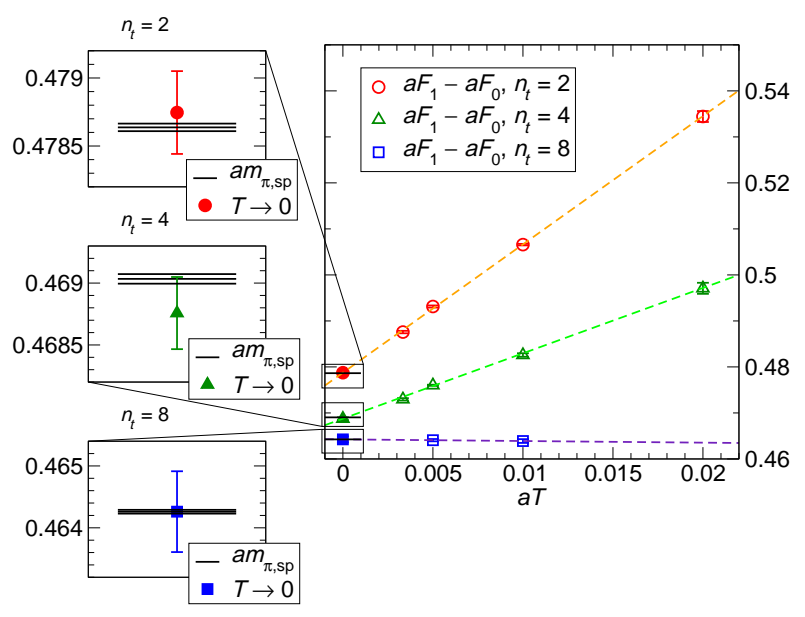

Figure 3: The differences of the free energies of the isospin one and isospin zero sectors as a function of the temperature on dynamical staggered configurations with a spatial volume of $6^{3}$. The dashed lines show the linear fits to the data points. The $T \rightarrow 0$ extrapolated values are compared to the spectroscopic pion masses.

\begin{tabular}{c|c}
\hline \hline $1 / a T$ & Number of configurations \\
\hline 48 & 2502 \\
96 & 1852 \\
192 & 731 \\
384 & 412 \\
\hline \hline
\end{tabular}

Table 3: Number of quenched configurations.

The r.h.s. of equation (47) does not explicitly contain the number of staggered tastes. The quantity

$$
\text { " } a F_{I_{3}=1}-a F_{I_{3}=0} "=-\frac{1}{L_{t}} \ln \left\langle\left|\sum_{k=1}^{3 V} \lambda_{k}\right|^{2}\right\rangle
$$

can be evaluated on quenched configurations as well. The question arises naturally: If one measures the pion mass on a quenched ensemble using regular staggered spectroscopy and evaluates the expression in (47) with the same fermion mass, will these be the same?

To find this out we performed calculations on quenched configurations generated using the Wilson plaquette gauge action. The spatial extension of the lattice was $L_{s}=6$, the gauge coupling was $\beta=5.6$ and the corresponding lattice spacing was $a=0.21 \mathrm{fm}$ [11]. The time extension of the used lattices were $L_{t}=48,96,192,384$ and for the measurements we used a bare quark mass of $a m_{q}=0.04$. The number of configurations used are listed in Table 3 . The results are summarized in Table 4 and the linear extrapolation is shown in Figure 4. The comparison shows that the result obtained from the free energies is consistent with the spectroscopic pion mass.

The partition function contains all the information about the degrees of freedom present in the system, therefore, the free energy should be able to make a difference between dynamical and quenched configurations. The results, however, show that both types of ensembles yield a free energy that is consistent with particles of mass equal to the spectroscopic pion mass present in the system. Thus, one cannot tell this way whether a given set of configurations is from a dynamical or a quenched ensemble.

\section{Conclusions}

We have proposed a spectroscopic method based on purely thermodynamical considerations. The formulae obtained show the relation between the eigenvalues of the reduced staggered fermion matrix and the hadron 


\begin{tabular}{c|c}
\hline \hline $1 / a T$ & " $a F_{I_{3}=1}-a F_{I_{3}=0} "$ \\
\hline 48 & $0.5393(6)$ \\
96 & $0.5389(2)$ \\
192 & $0.5385(3)$ \\
384 & $0.5389(4)$ \\
\hline$\rightarrow \infty$ & $0.5385(3)$ \\
\hline$a m_{\pi, \mathrm{sp}}$ & $0.53874(3)$ \\
\hline \hline
\end{tabular}

Table 4: The "differences of the free energies" of the isospin one and isospin zero sectors, their $T \rightarrow 0$ extrapolated value and the spectroscopic pion mass on quenched configurations.

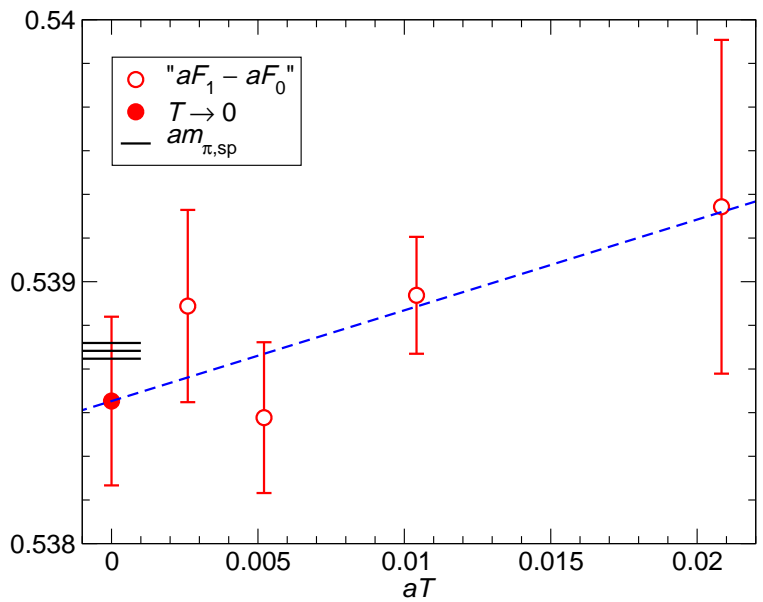

Figure 4: The "differences of the free energies" of the isospin one and isospin zero sectors as a function of the temperature on quenched configurations with a spatial volume of $6^{3}$ and bare quark mass $a m_{q}=0.04$. The dashed line shows the linear fit to the data points. The $T \rightarrow 0$ extrapolated value is compared to the spectroscopic pion mass. 
spectrum. The method not only clarifies the findings of Ref. [1] in connection with the Goldstone pion mass, but also extends them. In principle, the method can be used to obtain the mass of the lightest particle in a given quark number sector. For example, in principle, the mass of the di-baryon could be obtained. However, it turns out that the application even to one-baryon states is computationally very demanding. Nevertheless, we successfully applied our method to the Goldstone pion. In the calculations presented the mass of the lowest state in the $I_{3}=1$ sector is in agreement with the pion mass obtained using the ordinary spectroscopic method. This indicates that the method presented in Section 7 is a valid way of finding the pion mass.

\section{Acknowledgements}

We would like to thank G. I. Egri, C. Hoelbling and S. D. Katz for their help. This research was partially supported by OTKA Hungarian Science Grants No. T34980, T37615, M37071, T032501, AT049652 and by DFG German Research Grant No. FO 502/1-1. The computations were carried out on the 370 processor PC cluster of Eötvös University and on the 1024 processor PC cluster of Wuppertal University. We used a modified version of the publicly available MILC code [12] with next-neighbor communication architecture for PC-clusters [13].

\section{References}

[1] P. E. Gibbs, The fermion propagator matrix in lattice QCD, Phys. Lett. B172 (1986) 53.

[2] A. Alexandru, M. Faber, I. Horvath and K.-F. Liu, Lattice QCD at finite density via a new canonical approach, Phys. Rev. D72 (2005) 114513 [hep-lat/0507020].

[3] S. Kratochvila and P. de Forcrand, The canonical approach to finite density QCD, PoS LAT2005 (2006) 167 hep-lat/0509143.

[4] S. Kratochvila and P. de Forcrand, QCD at zero baryon density and the Polyakov loop paradox, Phys. Rev. D73 (2006) 114512 hep-lat/0602005].

[5] P. de Forcrand and S. Kratochvila, Finite density QCD with a canonical approach, Nucl. Phys. Proc. Suppl. 153 (2006) 62-67 hep-lat/0602024].

[6] A. Roberge and N. Weiss, Gauge theories with imaginary chemical potential and the phases of QCD, Nucl. Phys. B275 (1986) 734.

[7] P. Hasenfratz and F. Karsch, Chemical potential on the lattice, Phys. Lett. B125 (1983) 308.

[8] Z. Fodor and S. D. Katz, Lattice determination of the critical point of QCD at finite T and mu, JHEP 03 (2002) 014 hep-lat/0106002.

[9] A. Hasenfratz and D. Toussaint, Canonical ensembles and nonzero density quantum chromodynamics, Nucl. Phys. B371 (1992) 539-549.

[10] N. Weiss, The effective potential for the order parameter of gauge theories at finite temperature, Phys. Rev. D24 (1981) 475.

[11] R. G. Edwards, U. M. Heller and T. R. Klassen, Accurate scale determinations for the Wilson gauge action, Nucl. Phys. B517 (1998) 377-392 |hep-lat/9711003.

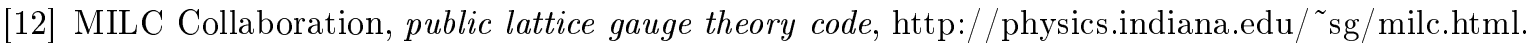

[13] Z. Fodor, S. D. Katz and G. Papp, Better than $\$ 1 /$ Mflops sustained: A scalable PC-based parallel computer for lattice QCD, Comput. Phys. Commun. 152 (2003) 121-134 [hep-lat/0202030]. 